\title{
The new UK Medical Eligibility Criteria (UKMEC): what has changed?
}

\section{Laura Percy}

ST6, Community Sexual and Reproductive Healthcare, New Croft Centre, Newcastle upon Tyne, UK

\section{Correspondence to} Dr Laura Percy, Sexual and Reproductive Healthcare, New Croft Centre, Market Street (East), Newcastle upon Tyne NE1 6ND, UK; laura.percy2@nuth.nhs.uk

Received 28 February 2016 Revised 3 March 2016 Accepted 3 March 2016

\section{CrossMark}

To cite: Percy L. J Fam Plann Reprod Health Care

2016;42:81-82.

\section{INTRODUCTION}

A welcome update of the UK Medical Eligibility Criteria (UKMEC) will be published in April 2016. ${ }^{1}$ It continues the UKMEC's role of providing guidance on the safety of contraceptive methods with regard to numerous medical conditions and patient characteristics. UKMEC 2016 is not designed to address the use of contraceptives for non-contraceptive indications (e.g. heavy menstrual bleeding). It does not consider the efficacy of a given method, nor the impact of the treatment for a given condition with regard to efficacy or drug interaction. Finally, it is not intended to replace clinical judgement. This editorial aims to provide an overview of new additions and notable changes to the UKMEC in relation to clinical practice. ${ }^{1}$

\section{REVISED FORMAT}

One of the most immediately notable features of the new UKMEC is the alteration in the order in which the methods of contraception are presented. The longacting reversible methods (LARC) are presented first, followed by medium- and then shorter-acting methods. This change clearly reflects the importance of promoting LARC use, particularly for women for whom pregnancy would pose a significant risk to their health due to their medical history (e.g. cystic fibrosis or complicated valvular heart disease). That said, the UKMEC continues to strenuously advocate that providing a woman is medically eligible to use a given method, she should be free to choose the option most acceptable to her. ${ }^{1}$

I would suggest that this change in method order will have the most significant impact on UKMEC users already familiar with the very useful summary table from the previous edition. Initially clinicians may find that the alterations to the layout will make using the new summary table more cumbersome than the older version. In time the new layout will become the norm and I would expect that it will then be found to be user-friendly.

\section{OMITTED OR MODIFIED SECTIONS}

Firstly, this update sees the removal of split categories, where more than one category is assigned to a given condition (e.g. $2 / 3$ or $3 / 4$ categories). ${ }^{2}$ This is a change that, I would anticipate, will be warmly welcomed by clinicians. ${ }^{1}$ Instead those conditions with previously split categories, such as viral hepatitis and diabetes, have been added to or expanded upon $^{1}$ to aid clinicians in making a more patient-centred judgement about the applicability of a method to a given individual.

Another notable change is that sterilisation and barrier methods have been removed from the $\mathrm{UKMEC}^{1}$ as they are comprehensively covered by methodspecific guidance produced by the Faculty of Sexual \& Reproductive Healthcare (FSRH). ${ }^{3} 4$

Furthermore, schistosomiasis and malaria have been removed ${ }^{1}$ as these are not common diseases in the UK and clinicians encountering these rarer conditions have recourse to the World Health Organization Medical Eligibility Criteria (WHOMEC). ${ }^{5}$ Finally, Raynaud's phenomenon has been removed, as expert opinion asserts that risk is associated with an underlying disease process rather than the phenomenon itself. In relation to this there has been simplification, and I think clarification, regarding the use of contraception in women with systemic lupus erythematosus. Decision making is now being based on the presence or absence of antiphospholipid antibodies. Furthermore, for those with positive antiphospholipid 
antibodies, progestogen-only methods are now a UKMEC 2, increasing ease of accessibly to contraception. ${ }^{1}$

\section{ADDITIONAL CONDITIONS}

There is the welcome addition of several conditions to this latest edition of the UKMEC including: history of bariatric surgery, organ transplant, cardiomyopathy, arrhythmias (AF and long QT syndrome) and rheumatoid arthritis. ${ }^{1}$ This reflects the increasing prevalence of women with these conditions requiring contraception and therefore the need to provide practitioners with guidance in their management.

\section{SEXUALLY TRANSMITTED INFECTIONS AND HIV}

Guidance pertaining to sexually transmitted infections has been altered, in that insertion of intrauterine contraception in a woman with asymptomatic chlamydial infection has been reduced from UKMEC 4 to UKMEC $3 .^{1}$ In addition, the guidance for contraceptive options in HIV infection has been expanded to incorporate CD4 count and no longer provides guidance in relation to those using antiretroviral medication. ${ }^{12}$

\section{POSTPARTUM CONTRACEPTION}

Guidance on postpartum contraception has also been amended. Regarding the use of combined hormonal contraception in breastfeeding women, the UKMEC now indicates that it is safe to use these methods in women over 6 weeks' postpartum (UKMEC 2, previously UKMEC 3). ${ }^{1} \quad{ }^{2} \quad$ Furthermore, for nonbreastfeeding women the categorisation is divided into those with and without risk factors for venous thromboembolism (VTE), wherein the risk factors are clearly specified. ${ }^{1}$ This change is another welcome addition, given the increased risk of VTE postpartum, it enables practitioners to more accurately present risks and benefits to clients.

\section{EMERGENCY CONTRACEPTION}

The new UKMEC also includes a section on emergency contraception (EC) and, following the theme of the document as a whole, the methods are presented in terms of effectiveness. As a result, the intrauterine device is presented before ulipristal acetate, both of which are then followed by levonorgestrel. This section sees the addition of obesity as a characteristic. It is a UKMEC 1 for all methods as data are too limited to conclude that increased body weight will reduce the effect of EC. ${ }^{1}$ In addition, there is expansion of advice in relation to gestational trophoblastic disease. This is now broken down into undetectable $\beta$-human chorionic gonadotropin ( $\beta$-hCG), decreasing $\beta$-hCG and persistently elevated $\beta$-hCG or malignant disease. ${ }^{1}$ This division further aids practitioners in helping patients make an appropriate decision with regard to EC.

\section{MIGRAINE}

Finally, I would anticipate that the addition of a resource on the diagnosis of migraine will prove to be a valuable tool for many practitioners. This additional resource provides a list of diagnostic criteria, fulfilment of which is required for the diagnosis of migraine and migraine with aura. ${ }^{1}$ The inclusion of this useful information will aid practitioners in making diagnostic decisions regarding the nature of headaches and therefore not unnecessarily restrict women in their contraceptive choices.

\section{CONCLUSION}

UKMEC 2016 remains essential and accessible. Caution may be needed as we begin to use this new version, given that the methods are presented in a different order. Overall, the authors of UKMEC 2016 have provided guidance that is thorough, robust and, most importantly, user-friendly.

Competing interests None declared.

Provenance and peer review Commissioned; internally peer reviewed.

\section{REFERENCES}

1 Faculty of Sexual \& Reproductive Healthcare. UK Medical Eligibility Criteria for Contraceptive Use (UKMEC 2016). 2016. http://www.fsrh.org/pdfs/UKMEC2016.pdf [accessed 2 April 2016]. (draft guidance to be published April 2016).

2 Faculty of Sexual \& Reproductive Healthcare. UK Medical Eligibility Criteria for Contraceptive Use (UKMEC 2009). 2009. http://www.fsrh.org/pdfs/UKMEC2009.pdf [accessed 17 February 2016].

3 Faculty of Sexual \& Reproductive Healthcare Clinical Effectiveness Unit. Male and Female Sterilisation. 2014. http:// www.fsrh.org/pdfs/MaleFemaleSterilisation.pdf [accessed 17 February 2016].

4 Faculty of Sexual \& Reproductive Healthcare Clinical Effectiveness Unit. Barrier Methods for Contraception and STI Prevention. 2012. http://www.fsrh.org/pdfs/ CEUGuidanceBarrierMethodsContraceptionSDI.pdf [accessed 17 February 2016].

5 World Health Organization. Medical Eligibility Criteria for Contraceptive Use (5th edn). 2015. http://www.who.int/ reproductivehealth/publications/family_planning/MEC-5/en/ [accessed 17 February 2016]. 